\title{
Clinical Effects of Pleurotomy on Postoperative Outcomes of Patients Undergoing On-Pump Coronary Artery Bypass Grafting with Skeletonized Left Internal Thoracic Artery
}

\author{
Emre Selçuk, ${ }^{1}$ Deniz Günay, ${ }^{2}$ Mehmet Aksüt, ${ }^{2}$ Tolga Baş, ${ }^{2}$ Hasan Erdem, ${ }^{2}$ Mehmet K. Kırali ${ }^{2}$ \\ ${ }^{1}$ Department of Cardiovascular Surgery, Bezmiâlem Foundation University, İstanbul, Turkey; \\ ${ }^{2}$ Department of Cardiovascular Surgery, Kartal Kosuyolu Research and Education Hospital İstanbul, Turkey
}

\section{ABSTRACT}

Purpose: To evaluate the clinical impact of pleurotomy during skeletonized internal thoracic artery (ITA) harvesting in patients undergoing on-pump coronary artery bypass grafting $(\mathrm{CABG})$.

Methods: Consecutive patients $(\mathrm{n}=758)$ who underwent CABG with skeletonized ITA were divided into 2 groups according to pleural integrity: open pleura $(\mathrm{OP})$ and closed pleura (CP). Propensity score matching was performed after retrospective data extraction. The measured outcomes were postoperative pulmonary and hemorrhagic complications, 30-day mortality, and duration of hospital stay.

Results: Among 236 propensity score-matched pairs, there was no statistically significant difference between the 2 groups in terms of first 30-day mortality (OP, $\mathrm{n}=7$ [3\%]; CP, $\mathrm{n}=5[2.5 \%])$, blood product use (OP, $0.90 \pm 0.71$; CP, 0.74 \pm 0.7 ), or median duration of hospital stay. The incidence of postoperative pleural effusion, thoracentesis, prolonged mechanical ventilation, respiratory failure, excessive drainage, cardiac tamponade, and reexploration and the number of patients requiring transfusion were similar in both groups.

Conclusion: The clinical effect of pleural protection or pleurotomy on postoperative outcomes is limited in patients undergoing on-pump CABG with skeletonized ITA.

\section{INTRODUCTION}

Pulmonary complications are a common problem after coronary artery bypass grafting (CABG) [Moazzami 2017]. Although its negative effect on pulmonary functions is common knowledge, the internal thoracic artery (ITA) is the gold standard in CABG because of its excellent graft patency [Neumann 2019]. For this reason, various ITA harvesting techniques have been compared by many authors,

Received August 31, 2020; accepted September 21, 2020.

Correspondence: Emre Selcuk, MD, Department of Cardiovascular Surgery, Bezmialem Foundation University Medical Faculty Hospital, Yah Mah., Sabil Yolu Sk. No:16, 34844 Maltepe/Istanbul, 34844 Turkey; 905057233151 (e-mail:dr:emreselcuk@gmail.com). especially in terms of pulmonary effects and bleeding. It has been emphasized in several studies that pleural preservation reduces respiratory complications and decreases bleeding risk [Ali-Hassan-Sayegh 2014]. Pleurotomy for ITA harvesting is frequently used in daily practice, however, because it allows a longer time for graft harvesting and theoretically reduces the risk of pericardial tamponade. Additionally, reproducibility of the closed pleural technique is low in some patients because of anatomy and is closely dependent on operator experience.

Another ITA harvest modification is the skeletonized technique. Skeletonization of the ITA facilitates harvesting of a longer conduit and allows for sequential anastomosis [Sun 2015]. It has been speculated that, with skeletonized ITA harvest, the frequency of sternal problems is lower than with the pedicled technique, owing to better preservation of intracostal circulation. Postoperative hemorrhagic complications and sternal pain are reduced as well [Kamiya 2018; Ozzülkü 2016].

The effects of pleural preservation have been well studied in the pedicled left internal thoracic artery (LITA) harvesting technique, but only a few studies have evaluated the effects of pleurotomy in the skeletonized technique. In this study, we investigated the effects of pleural preservation on clinical outcomes in terms of respiratory and hemorrhagic complications in patients who underwent CABG with skeletonized ITA.

\section{MATERIALS AND METHODS}

\section{Study Population and Data Source}

This multisurgeon, single-center retrospective study included all patients who underwent on-pump CABG (skeletonized LITA for left anterior descending artery) between January 2013 and January 2018 at a high-level cardiac surgery center.

Exclusion criteria were (1) off-pump CABG, (2) use of bilateral internal thoracic artery (BITA), (3) nonskeletonized techniques for ITA harvesting (pedicled or semiskeletonized), (4) previous cardiac surgery, (5) concomitant cardiac procedures, and (6) intraoperative mortality.

Patients were divided into 2 groups (OP, open pleura; $\mathrm{CP}$, closed pleura) based on pleural integrity during LITA harvest. Early postoperative outcomes analyzed the pros and cons of pleural preservation. Demographic and clinical information of the patients were obtained from hospital records. The 
study was conducted in accordance with the Helsinki Declaration after the approval of the institutional ethical committee (protocol number 2018.4/2-134).

\section{Procedural Characteristics}

The decision of surgical revascularization was made by the consensus of $\geq^{1}$ cardiac surgeon and a cardiologist. Surgical revascularization was performed with conventional on-pump CABG technique (LITA for left anterior descending artery and saphenous vein for other vessels).

In all study patients, LITA harvesting was performed using the skeletonization technique by a single harvester. After median sternotomy, the parietal pleura was gently dissected from the chest wall with electrocautery. The second step was incision of the endothoracic fascia along the medial side of LITA. Pulling down the fascia provided direct vision of LITA and accompanying veins. LITA was isolated from the endothoracic fascia and muscles, preserving venous structures through the proximal portion to the bifurcation of the vessel. Because of its delicate vessel structure, LITA was never directly grabbed with forceps. Side branches of LITA were ligated with small-sized vascular clips on both sides, then divided with scissors. Systemic heparinization was performed after meticulous dissection was completed, and LITA was clipped at the level of its distal portion. After visually confirming that graft flow was adequate, the graft was wrapped with papaverine-soaked gauze.

No systemic institutional opinion exists related to pleural presentation or pleurotomy for LITA harvest. Therefore, it was the harvester's decision whether to open the pleural cavity. Of note, if the pleural cavity was unintentionally opened in the closed pleural technique, it was opened completely, rather than undergoing repair. A left thorax drain (32 F) and a mediastinal drain $(28 \mathrm{~F})$ were routinely placed in OP group patients. In $\mathrm{CP}$ group patients, a retrocardiac drain was placed in addition to a mediastinal drain. All patients were managed postoperatively with the same standardized institutional protocols.

Chest tubes were pulled if the latest 8 hours of drainage were $<100 \mathrm{cc}$ without air leak. Chest $\mathrm{x}$-rays on day 6 after surgery, the first week after discharge, and the first month after discharge were examined for pleural effusion. If the patient had any history of thoracentesis, prethoracentesis lung film was also checked.

\section{Study Variables and Analysis of Outcomes}

Demographic characteristics of the patients (age, sex, and body mass index [BMI]), concomitant diseases (diabetes mellitus, hypertension, smoking, chronic obstructive pulmonary disease [COPD], extracardiac arteriopathy, and chronic kidney disease), left ventricular (LV) dysfunction (ejection fraction $<40 \%$ ), operative priority (emergent, urgent, or elective), preoperative antiplatelet therapy, and intraoperative variables (cross-clamp time, cardiopulmonary bypass [CPB] time, and number of grafts) were evaluated. Pleural effusions, thoracentesis, prolonged mechanical ventilation, respiratory failure, excessive drainage $(>1000 \mathrm{~mL})$, cardiac tamponade, reexploration, need for blood transfusions, and duration of hospital stay were compared between the 2 groups.
Prolonged mechanical ventilation was defined as requiring mechanical ventilation for $>24$ hours at any time in the postoperative period. Prolonged mechanical ventilation, reintubation because of hypoxia or hypercarbia during hospitalization, or the need for persistent noninvasive mechanical ventilation support was accepted as postoperative respiratory failure.

\section{Statistical Analysis}

Because of lack of randomization with regard to pleural preservation, a propensity score (PS) was generated for each patient from a multivariable logistic regression model based on preoperative and intraoperative covariates as independent variables, with $\mathrm{OP}$ versus $\mathrm{CP}$ techniques as a binary dependent variable. Covariates included in the PS model were age, female sex, BMI, diabetes, extracardiac arteriopathy, hypertension, smoking, COPD, chronic kidney disease, LV dysfunction, graft number, CPB time, cross-clamp time, elective surgery, operative priority [urgent, emergent, elective], and preoperative antiplatelet therapy [none, acetylsalicylic acid [ASA], clopidogrel, dual antiplatelet]). Pairs of patients were derived by nearest-neighbor 1:1 matching with a caliper width of 0.1 standard deviation (SD) of the logit of the PS. Matching was performed using the MatchIt package; covariate balance was assessed using Cobalt; and balance improvements are presented using the love.plot command [Ho 2011; Greifer 2020; Imai 2014].

Categorical variables are presented as counts and frequencies, and continuous variables as mean (SD) or median (interquartile range $[\mathrm{IQR}]$ ), as appropriate. Fisher's exact test or $\chi^{2}$ test was used for comparison between categorical variables. Student's $t$ test or Mann-Whitney $U$ test was used to compare continuous variables. A 2-tailed $P$ value of 0.05 was considered statistically significant. R Statistical Software (version 3.6.3; R Foundation for Statistical Computing, Vienna, Austria) was used for all statistical analyses.

\section{RESULTS}

A total of 758 consecutive patients who met inclusion criteria were assessed. Patients were stratified according to pleural integrity. OP was present in $71.1 \%(\mathrm{n}=547)$ and $\mathrm{CP}$ in $28.9 \%(n=251)$ of the study population. Female sex, elective operation, and preoperative ASA usage were much more common in the CP group. The BMI of patients in the OP group was higher than in $\mathrm{CP}$. The number of patients with LV dysfunction was higher in the OP group. After PS matching, 2 groups of 236 patients were obtained. Balance improvement after the propensity match is illustrated in the Figure. The 2 groups were comparable for all pretreatment variables investigated after matching. Table 1 presents the variables of the patients.

The first 30-day lethal outcome ratio was $2.5 \%(\mathrm{n}=12)$. There was no statistically significant difference between the 2 groups in terms of mortality in the first 30 days (OP, $\mathrm{n}=7$ [3\%]; CP, $\mathrm{n}=5[2.5 \%])$. A mean $( \pm \mathrm{SD})$ of $0.84 \pm 0.5$ units of blood products per patient was used, with no significant 


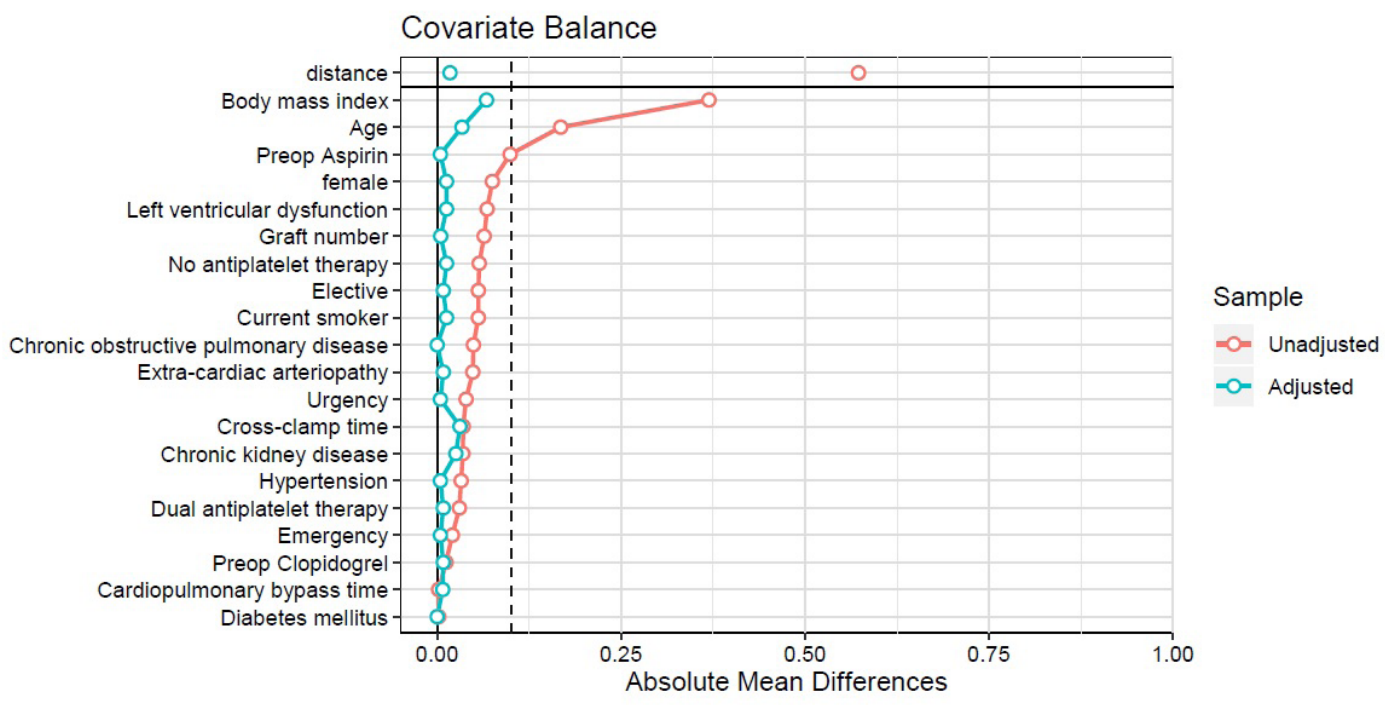

Figure. Balance improvements for baseline variables before and after PS matching

Table 1. Baseline Characteristics of the Study Population*

\begin{tabular}{|c|c|c|c|c|c|c|c|c|}
\hline \multirow[b]{2}{*}{ Variable } & \multicolumn{2}{|c|}{ Before Match } & \multicolumn{4}{|c|}{ After Match } & \multicolumn{2}{|c|}{ SMD } \\
\hline & OP & $\mathrm{CP}$ & $P$ Value & OP & $\mathrm{CP}$ & $P$ Value & Before & After \\
\hline Patients & 547 & 251 & & 236 & 236 & & & \\
\hline Female sex & $142(26)$ & $84(33.5)$ & .02 & $63(25.9)$ & $72(29.6)$ & .362 & 0.0751 & 0.0127 \\
\hline Age $(y)$ & $58(13)$ & $62(10)$ & .66 & $61(18)$ & $61(17)$ & .67 & 1.4808 & -0.2966 \\
\hline $\mathrm{BMI}(\mathrm{kg} / \mathrm{m} 2)$ & $27.8(5.3)$ & $27.1(4.2)$ & .009 & $28.7(7.4)$ & $28.5(5)$ & .47 & -1.2980 & -0.2352 \\
\hline Extracardiac arteriopathy & $65(11.9)$ & $42(16.7)$ & .062 & $41(16.9)$ & $38(15.6)$ & .712 & 0.0485 & 0.0085 \\
\hline Hypertension & $84(33.5)$ & $201(36.7)$ & .36 & $120(49.4)$ & 119 (49) & .92 & -0.0328 & -0.0042 \\
\hline Current smoker & $218(39.9)$ & $86(34.3)$ & .13 & $100(41.2)$ & $105(43.2)$ & .64 & -0.0559 & 0.0127 \\
\hline COPD & $88(16.1)$ & $28(11.2)$ & .66 & $28(11.5)$ & $26(10.7)$ & .77 & -0.0493 & 0.0000 \\
\hline CPB time (min) & $95(30)$ & $97(23)$ & .64 & $50(19)$ & $47(21)$ & .42 & 0.7326 & 0.6398 \\
\hline Cross-clamp time (min) & $50(19)$ & $47(21)$ & .62 & $95(28)$ & $97(23)$ & .46 & 0.0530 & 0.1864 \\
\hline Elective & $475(86.8)$ & $232(92.4)$ & .021 & $223(91.8)$ & $217(89.3)$ & .35 & 0.0559 & -0.0085 \\
\hline Urgent & $52(9.5)$ & $14(5.6)$ & .61 & $19(7.8)$ & $22(9.1)$ & .624 & -0.0393 & 0.0042 \\
\hline Emergent & $20(3.7)$ & $4(1.6)$ & .11 & $1(0.4)$ & $4(1.6)$ & .17 & -0.0206 & 0.0042 \\
\hline No antiplatelets & $367(67.1)$ & $154(61.4)$ & .11 & $158(65)$ & $149(61.3)$ & 1.00 & -0.0574 & -0.0127 \\
\hline ASA & $133(24.3)$ & $86(34.3)$ & .003 & $76(31.3)$ & $84(34.6)$ & 1.00 & 0.0995 & -0.0042 \\
\hline Clopidogrel & $24(4.4)$ & $8(3.2)$ & .42 & $7(2.9)$ & $7(2.9)$ & 1.00 & -0.0120 & 0.0085 \\
\hline DAPT & $23(4.2)$ & $3(1.2)$ & .26 & $2(0.8)$ & $3(1.2)$ & .65 & -0.0301 & 0.0085 \\
\hline
\end{tabular}

*Data are $\mathrm{n}, \mathrm{n}(\%)$, or median (IQR).

DAPT indicates dual antiplatelet therapy; SMD, standardized mean difference. 
difference between the 2 groups $(\mathrm{OP}, 0.90 \pm 0.71$; $\mathrm{CP}, 0.74 \pm$ $0.7)$. The incidence of postoperative pleural effusion, thoracentesis, prolonged mechanical ventilation, respiratory failure, excessive drainage, cardiac tamponade, and reexploration and the number of patients requiring transfusion were similar in both groups. The postoperative results of the 236 PSmatched pairs are presented in Table 2 .

\section{DISCUSSION}

This study examined the relationship between preserving pleural integrity and postoperative respiratory and hemorrhagic complications in patients undergoing conventional CABG with skeletonized ITA. We found that pleural preservation did not have a statistically significant clinical effect in terms of postoperative hemorrhagic or respiratory complications during skeletonized ITA harvesting.

Pulmonary impairment is an inevitable result of openheart surgery. Sternotomy, fluid shift, hemodynamic changes, general anesthesia, blood product usage, postoperative pain, systemic inflammatory response due to cardiopulmonary bypass, postoperative pleural effusion, and atelectasis are potential causes of pulmonary damage [Huffmyer 2015; Ng 2002]. Additionally, several studies have emphasized that pleurotomy during pedicled ITA harvesting is associated with poor postoperative outcomes compared with pleural preservation [Guizilini 2007; Ghavidel 2013; Atay 2009]. Increased postoperative atelectasis and pleural effusion rate associated with pleurotomy have been reported during ITA harvest [Atay 2009; Oz 2006]. Oz et al [2006] reported that the postoperative multidimensional pain score was higher in patients whose pleural integrity was not preserved versus others in their prospective randomized study. In some studies, postoperative bleeding and transfusion requirements have been shown to be higher in patients with opened pleura [Atay 2009; Iskesen 2009].

Contrary to consecutive reports about the negative clinical effects of pleurotomy in the pedicled ITA harvest technique, we found no statistically significant difference in pleural integrity in terms of pulmonary complications or postoperative bleeding in patients undergoing CABG with skeletonized LIMA. Bonacci et al [2001], in their study comparing 3 different ITA harvesting techniques for bilateral ITA (onpump $\mathrm{CABG}$ ), found that early postoperative pain in $\mathrm{OP}$ patients was significantly higher than in $\mathrm{CP}$ patients. The authors emphasized that blood gas parameters were similar after removal of the thorax tube. Pleural effusions were much higher in the OP group than in the CP group. An important limitation of the study was the small number of OP patients $(n=30)$ [Bonacci 2001]. Guizilini et al [2007] reported that pleurotomy was associated with increased pulmonary dysfunction in a prospective study of on-pump CABG using skeletonized LITA for left anterior descending artery (LAD) (15 patients in each group). A later study that included a larger group of patients who underwent $C A B G$ with skeletonized ITA, with preferred subxiphoid pleural drain, showed similar clinical outcomes in the 2 patient groups [Guizilini 2012].

Skeletonized LITA harvesting is characterized by a reduced incidence of sternal complications, respiratory complications, and postoperative pain compared with the pedicled technique [Fouquet 2015]. In some series, patients with skeletonized ITA have been shown to have reduced postoperative drainage [Özülkü 2016; Calafiore 1999]. Skeletonized LITA harvesting requires more surgical experience and more meticulous dissection than the pedicle technique, aside from all other pros and cons. We could speculate that skeletonization minimizes the previously mentioned negative effects of pleural violation, according to the results of this study and the current literature.

Some surgeons routinely open the pleural cavity to reduce the risk of tamponade and to avoid potential ITA tension during inflation, and sometimes just for better exposure [Ali 1996]. In our study, the tamponade rate was slightly higher in CP patients, but the difference was not significant. We definitely put extra drains-preferably retrocardiac drains-in $\mathrm{CP}$ patients. Additionally, we rarely experience problems with ITA tension, as skeletonization allows for long graft harvest.

Table 2. Postoperative Clinical Outcomes*

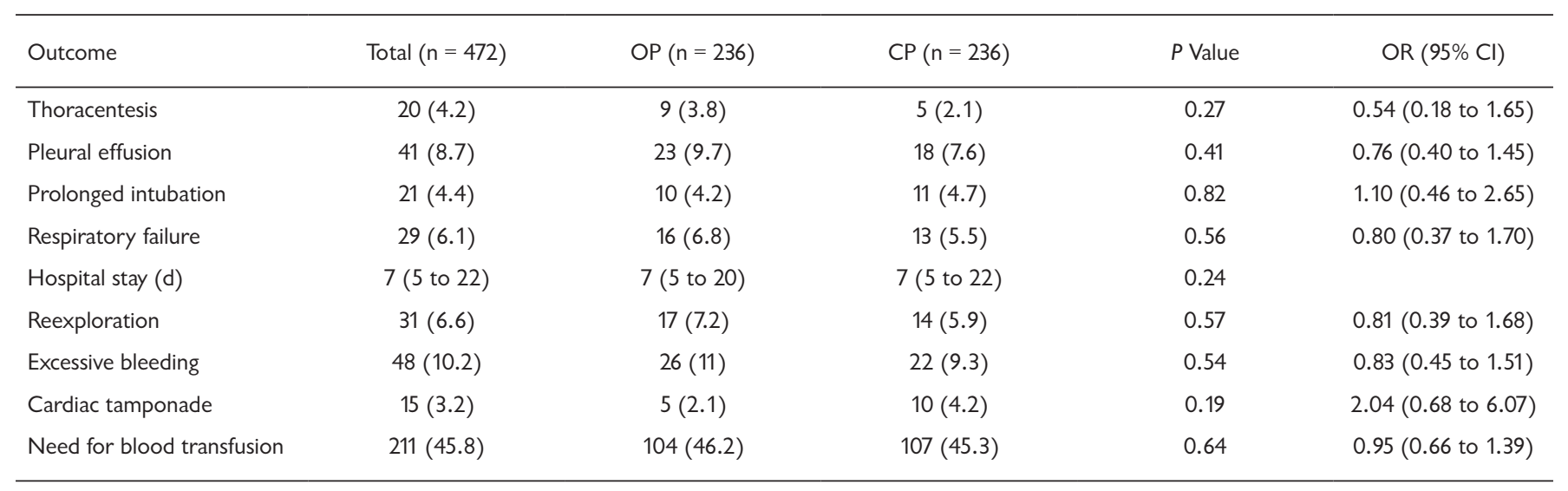

Data are $\mathrm{n}(\%)$ or median (minimum, maximum). 
Many studies on the effects of pleurotomy during ITA harvesting have been published, but the present study differs from previous studies in several ways. We have not seen any other report that includes isolated on-pump skeletonized LITA-LAD bypass patients. We kept the exclusion criteria very limited, unlike many other studies. In previous studies, the authors tended to exclude emergent or urgent operations or patients with chronic lung disease, preoperative platelet therapy, or diabetes from the study population to ensure standardization [Ali-Hassan-Sayegh 2014]. We included these patients because we believe they are an important part of surgical practice.

\section{Limitations}

This study has some limitations, primarily due to its retrospective design. Because the reproducibility of pleural protection was low in some patients, we could not perform healthy randomization. Although we made robust matching to limit the effects of patient-based variables, it is possible that surgeon-related bias might still exist. Consequently, we did not find a statistically significant difference between pleural protection and pleurotomy in patients who underwent CABG using skeletonized LITA.

\section{Conclusion}

The case-based liberal approach, according to surgeons' experience, seems acceptable as a pleural protection strategy during skeletonized LITA harvest.

\section{ACKNOWLEDGMENTS}

All of the authors were working at the reference hospital during the study period.

\section{REFERENCES}

Ali IM, Lau P, Kinley CE, Sanalla A. Opening the pleura during internal mammary artery harvesting: Advantages and disadvantages. Can J Surg 1996;39:42.

Ali-Hassan-Sayegh S, Mirhosseini SJ, Vahabzadeh V, Ghaffari N. Should the integrity of the pleura during internal mammary artery harvesting be preserved? Interact Cardiovasc Thorac Surg 2014;19:838-847.

Atay Y, Yagdi T, Engin C, et al. Effect of pleurotomy on blood loss during coronary artery bypass grafting. J Cardiac Surg 2009;24:122-126.

Bonacchi M, Prifti E, Giunti G, Salica A, Frati G, Sani G. Respiratory dysfunction after coronary artery bypass grafting employing bilateral internal mammary arteries: The influence of intact pleura. Eur J Cardiothorac Surg 2001;19:827-833.
Calafiore AM, Vitolla G, Iaco AL, et al. Bilateral internal mammary artery grafting: Midterm results of pedicled versus skeletonized conduits. Ann Thorac Surg 1999;67:1637-1642.

Fouquet O, Tariel F, Desulauze P, Mével G. Does a skeletonized internal thoracic artery give fewer postoperative complications than a pedicled artery for patients undergoing coronary artery bypass grafting? Interact Cardiovasc Thorac Surg 2015;20:663-668.

Ghavidel AA, Noorizadeh E, Pouraliakbar H, et al. Impact of intact pleura during left internal mammary artery harvesting on clinical outcome. J Tehran Univ Heart Center 2013;8:48.

Greifer, N. Cobalt: Covariate balance tables and plots. R package version 4.1.0. 2020. Available at: https://cran.rproject.org/web/packages/cobalt/ vignettes/cobalt_A0_basic_use.Htm l\#fn1

Guizilini S, Gomes WJ, Faresin SM, et al. Influence of pleurotomy on pulmonary function after off-pump coronary artery bypass grafting. Ann Thorac Surg 2007;84:817-822.

Guizilini S, Bolzan DW, Faresin SM, et al. Pleurotomy with subxyphoid pleural drain affords similar effects to pleural integrity in pulmonary function after off-pump coronary artery bypass graft. J Cardiothorac Surg 2012;7:1-7.

Ho D, Imai K, King G, Stuart EA. MatchIt: Nonparametric preprocessing for parametric causal inference. J Stat Softw 2011;42:1-28.

Huffmyer JL, Groves DS. 2015. Pulmonary complications of cardiopulmonary bypass. Best Pract Res Clin Anaesthesiol 2007;29:163-175.

Imai K, Ratkovic M. Covariate balancing propensity score. J R Stat Soc Ser B Stat Methodol 2014;76:243-263.

Iskesen I, Kurdal AT, Yildirim F, Cerrahoglu M, Sirin H. Pleura preservation during coronary operation does not have any beneficial effect on respiratory functions. Minerva Chir 2009;64:419-425.

Kamiya H, Akhyari P, Martens A, Karck M, Haverich A, Lichtenberg A. Sternal microcirculation after skeletonized versus pedicled harvesting of the internal thoracic artery: A randomized study. J Thorac Cardiovasc Surg 2018;135:32-37.

Moazzami K, Dolmatova E, Maher J, et al. In-hospital outcomes and complications of coronary artery bypass grafting in the United States between 2008 and 2012. J Cardiothorac Vasc Anesth 2017;31:19-25.

Neumann FJ, Sousa-Uva M, Ahlsson A, et al. 2018 ESC/EACTS Guidelines on myocardial revascularization. Eur Heart J 2019;40:87-165.

$\mathrm{Ng}$ CS, Wan S, Yim AP, Arifi AA. Pulmonary dysfunction after cardiac surgery. Chest 2002;121:1269-1277.

Oz BS, Iyem H, Akay HT, et al. Preservation of pleural integrity during coronary artery bypass surgery affects respiratory functions and postoperative pain: a prospective study. Can Respir J 2006;13:145-149.

Özülkü M, Aygün F. Effect of LIMA harvesting technique on postoperative drainage in off-pump CABG. Braz J Cardiovasc Surg. Braz J Cardiovasc Surg 2016;31:120-126.

Sun X, Huang J, Wang W, et al. Off-pump skeletonized versus pedicled left internal mammary artery grafting: Mid-term results. J Cardiac Surg 2015;30:494-499. 